\title{
Glucose Diffusion Limiting Membrane Based on Polyethyleneimine (PEI) Hydrogel for the Stabilization of Glucose Sensor
}

\author{
Suk-Joon Kim ${ }^{1}$ and Woonsup Shin ${ }^{1,2 *}$ \\ ${ }^{1}$ Department of Chemistry, Sogang University, Seoul 121-742, Korea \\ ${ }^{2}$ Department of Biomedical Engineering, Sogang University, Seoul 121-742, Korea
}

\begin{abstract}
Commercially available continuous glucose sensors require the operation stability for more than two weeks. Typically, the sensor comprises a sensing layer and an over-coating layer for the stable operation inside the body. In the sensing layer, enzymes and mediators are cross-linked together for the effective sensing of the glucose. The over-coating layer limits the flux of glucose and works as a biocompatible layer to the body fluids. Here, we report the simple preparation of the fluxlimiting layer by the condensation of polyethyleneimine (PEI), tri-epoxide linker, and trimethylolpropane triglycidyl ether (PTGE). The sensor is constructed by a layer-by-layer drop-coating of the sensing layer containing glucose dehydrogenase and the PEI-derived blocking layer. It is stable for more than 14 days, which is enough for the sensor in the continuous monitor glucose monitoring (CGM) system.
\end{abstract}

Keywords : Continuous Glucose Monitoring System (CGMS), Enzymatic Glucose Sensor, Diffusion Limiting Membrane

Received : 5 October 2020, Accepted : 20 November 2020

\section{Introduction}

Continuous glucose monitoring (CGM) system has been developed to efficiently monitor glucose level change in the body fluid of the people with diabetes $[1,2,3]$. In case of the disposable single-use strip sensor, it is difficult to monitor the change of blood glucose level because, in each measurement, it requires the sampling of capillary blood from the fingertip. On the other hand, CGM is inserted into the subcutaneous region, and it measures the glucose level in the interstitial fluid (ISF) continuously. The glucose level in the ISF is in good correlation with the blood glucose level $[4,5]$. Currently, the CGM replaces the disposable sensor rapidly in the diabetes market, and Dexcom, Abbott Diabetes, and Medtronic are the major players. The CGM sensor usually operates more than a week, and the stability of the glucose sensitivity is the most crucial requirement. For the purpose, an over-coat membrane exists to increase

*E-mail address: shinws@sogang.ac.kr

DOI: https://doi.org/10.33961/jecst.2020.01487

This is an open-access article distributed under the terms of the Creative Commons Attribution Non-Commercial License (http://creativecommons.org/licenses/by-nc/4.0) Attribution Non-Commercial License (http://creativecommons.org/licenses/by-nc/4.0)
which permits unrestricted non-commercial use, distribution, and reproduction in any which permits unrestricted non-commercial use, distri
medium, provided the original work is properly cited. the bio-compatibility and limit the glucose flux [6].

For the over-coating layer, the polymer-based membrane is generally in use, such as cellulose acetate $[7,8]$, conducting polymers $[9,10]$, xerogels $[11,12]$, sulfonated polymers like Nafion ${ }^{\circledR}[13,14,15]$, hydrophilic polyurethane $[16,17,18,19]$, chitosan $[20,21,22]$, etc. For example, in the Freestyle Navigator CGM sensor from Abbott, a cross-linked polyvinylpyridine (PVP) derivative is employed for the overcoating layer [6]. It is assumed that the currently commercially available CGM sensors, which claims the stability of one to two weeks, are using polyurethane based polymers for Dexcom sensors and polyvinylpyridine or polyvinylimidazole based polymers for Abbot sensors. But the actual structure and composition are hidden.

The normal glucose level in blood and interstitial fluid is about $5 \mathrm{mM}$ concentration and, if it is measured directly by the amperometric enzyme sensor, the current is in the range of at least uA level. However, for the long-term operation in the body, $\mathrm{nA}$ level current is essential for the system to be operated more than a week with a small coin-type battery. The low-current operation also helps the system stable by minimizing the generation of the products and bi- 
products. In glucose sensing, the decrease in the flux reduces the amounts of catalytic reaction products such as gluconic acid and hydrogen peroxide. The factors that affect the flux of glucose can be a charge, porosity, and the thickness of the membrane.

In this work, we introduced a new overcoating layer comprising, commercially available, a branched-polyethyleneimine (PEI) cross-linked with tri-epoxide linker, trimethylolpropane triglycidyl ether (PTGE). The glucose sensitivity was stable for more than two weeks for the sensor having the overcoated layer. The preparation of the PEI-based layer is much more simple and inexpensive compared to the other previously reported coating-layers.

\section{Experimental}

\subsection{Reagents and chemicals}

Sodium chloride, potassium chloride, disodium phosphate, and monopotassium phosphate were purchased from Alfa Aesar. D-glucose, glucose dehydrogenase $(\mathrm{GDH})$, ethanol $(\mathrm{EtOH})$, polyethyleneimine (PEI, Mw 600), trimethylolpropane triglycidyl ether (PTGE), and poly(ethylene glycol) diglycidyl ether (PEGDGE, Mw 500) were purchased from SigmaAldrich. Deionized water was from Purelab option Q. Polyvinylimidazole-Os(bipyridine) ${ }_{2}$ chlroride [PVI$\left.\mathrm{Os}(\mathrm{bpy})_{2} \mathrm{Cl}\right]$ was synthesized based on the literature [23].

\subsection{Fabrication of glucose sensor}

2.2.1 GDH/Os polymer/PEGDGE sensing layer

Glucose dhydrogenase(GDH), PVI-Os(bpy) ${ }_{2} \mathrm{Cl}$, and PEGDGE were prepared in 40,5 , and $10 \mathrm{mg} / \mathrm{mL}$ in water, respectively. A cocktail solution for coating was prepared by mixing PVI-Os(bpy $)_{2} \mathrm{Cl}$ : GDH: PEGDGE to be a mass ratio of $46: 42: 6$. The final concentration of the cocktail is $4.5 \mathrm{mg} / \mathrm{mL}$. Then, $5 \mathrm{uL}$ of the solution was drop-coated onto an air plasma-treated screen carbon printed electrode (SPCE, $5 \mathrm{~mm}$ diameter, area $=20 \mathrm{~mm}^{2}$ ) and dried for one day at an ambient condition.

\subsubsection{PEI-based diffusion limiting layer}

PEI and PTGE were prepared at $10 \mathrm{mg} / \mathrm{mL}$ in EtOH separately and mixed at a weight ratio of $1: 1$. Then, an appropriate amount of the mixed solution was drop-coated onto the sensing layer and dried for one day. The thickness of the over-coated layer made by drocoating $5 \mathrm{uL}$ of the solution is estimated to be 4 um [24].

\subsection{Electrochemical analysis}

The CHI 1230A potentiostat was used for the measurements of cyclic voltammetry and amperometry. The electrochemical measurements were performed by a three-electrode system, the working for glucose sensing, Pt counter, and $\mathrm{Ag} / \mathrm{AgCl}$ reference electrodes in PBS (phosphate buffer saline, $\mathrm{pH}$ 7.4).

\section{Results and Discussion}

Branched polyethyleneimine (PEI) is rich in amines, and trimethylolpropane triglycidyl ether (PTGE) contains terminal epoxides, as shown in Fig. 1. Therefore, the crosslinking between amines and epoxides can make a high-molecular-weight random copolymer, which can be insoluble in water.

The electrochemical properties of the sensing layer and the overcoated layer are shown in Fig. 2. For comparison, three kinds of sensors were prepared. First, the sensing layer only, A, was made by dropcoating $5 \mathrm{uL}$ of the cocktail solution of GDH, Os polymer, and PEGDGE in 42:46:6 ratio. For an overcoated sensor, B1, the PEI and PTGE mixed solution was drop-coated twice in $5 \mathrm{uL}$ onto the sensing layer. For another over-coated sensor, B2, the overcoating solution was drop-coated four times in $5 \mathrm{uL}$. a)<smiles>CCN(CCN)CCNCCN(CCNCCN)CCN(CCN)CCN(CCN)CCN</smiles>

b)

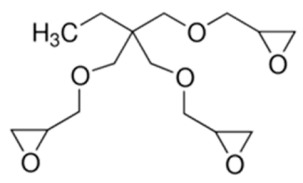

Fig. 1. Molecular structure of PEI (a) and PTGE (b). 
In case of electrode $\mathrm{A}$, it shows a typical reversible behavior of the Os(II)/Os(III) redox reactions of the redox polymer at formal potential, $\mathrm{E}^{0^{\prime}}=0.25 \mathrm{~V}$ vs. $\mathrm{Ag} / \mathrm{AgCl}$. Upon the coating of the PEI/PTGE layer (the electrode B1), the current decreased by about $20 \%$, and the $\mathrm{E}^{0}$ value shifted positively by $60 \mathrm{mV}$ compared to the electrode A. Further coating of PEI/ PTGE (the electrode B2) showed no significant difference compared to the electrode B1. The decrease of the current and the potential shift can be explained by the retardation of the transport of the chloride anion by the overcoating layer. It is known that chlo-

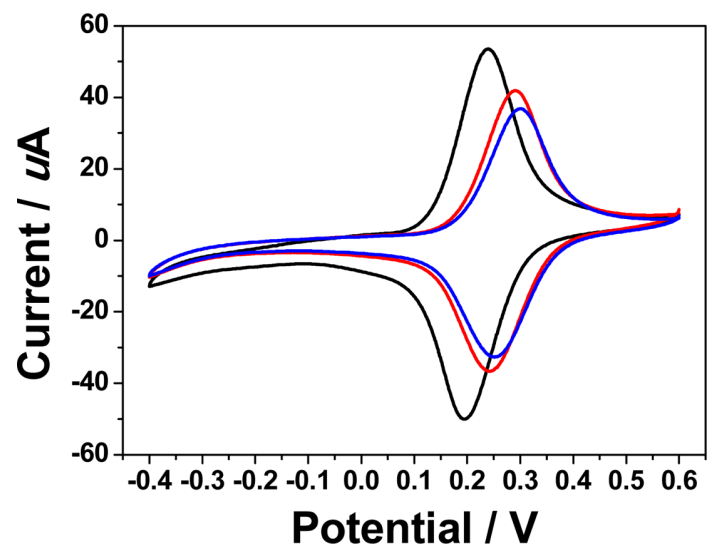

Fig. 2. $\mathrm{CVs}$ before and after introducing PEI/PTGE overcoating layer to the sensing layer in PBS at $10 \mathrm{mV} / \mathrm{s}$. (a) the electrode A; sensing layer only (black line), (b) the electrode B1; the sensor with the overcoated layer (red line), (c) the electrode B2; the sensor with the twice amount of the overcoated layer than B1 (blue line), as described in the text. ride transport is the limiting factor in the redox reactions of the osmium redox polymers [25]. Note that the PEI/PTGE layer can be regarded as an anion exchanger since it has a lot of amine functional groups.

The glucose sensitivity was measured by the amperometry for the three electrodes (Fig. 3). The glucose concentration was changed by the incremental addition of $1 \mathrm{M}$ glucose solution from 0 to $5 \mathrm{mM}$.

The current response, which is a glucose response, dropped significantly upon the coating of PEI/PTGE layer; $1 / 10$ for the electrode B1, 1/50 for the electrode B2. It can be explained by the significant retardation of glucose transport through the PEI/PTGE layer, although the transport of the chloride is affected by only $20 \%$ (See CVs in Fig. 2). The glucose response is still linear to the concentration for the coated layers, although the sensing current decreased significantly. For both electrode B1 and B2, the response to the glucose was linear in the range of 1 to $5 \mathrm{mM}$. Therefore, it is evident that the glucose concentration could be measured, although the sensitivity decreased by a factor of $1 / 50$ in case of the electrode B2. It showed the promise to be utilized in the body fluid, such as ISF.

We tested the long-term stability of the PEI/PTGE coated sensor in a $5 \mathrm{mM}$ glucose solution, which is the normal glucose concentration in the blood. The amperometric response at $0.35 \mathrm{~V}$ vs. $\mathrm{Ag} / \mathrm{AgCl}$ was monitored continuously for 26 days, and it showed the current response was stable over 20 days, as shown in Fig. 4.

For comparison, the response of the electrode hav-

a)

b)

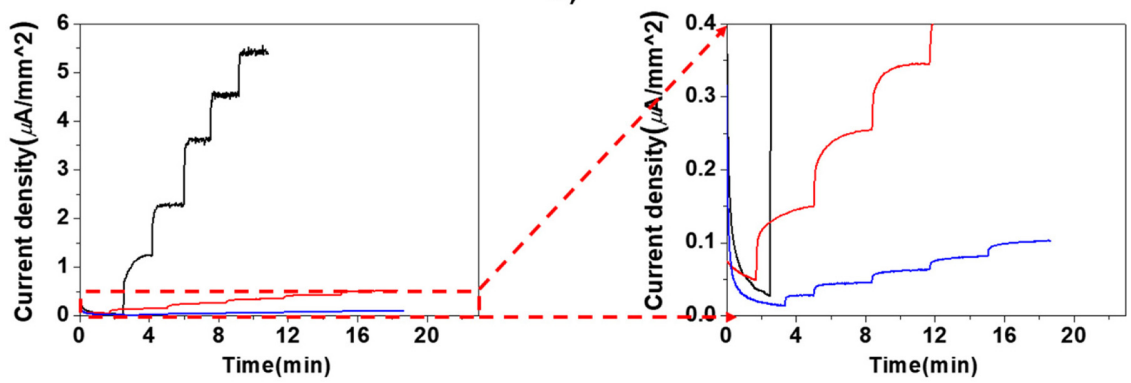

Fig. 3. Amperometric response at $0.35 \mathrm{~V}$ vs. $\mathrm{Ag} / \mathrm{AgCl}$, before and after introducing $\mathrm{PEI} / \mathrm{PTGE}$ layer. The glucose concentration was changed from $1 \mathrm{mM}$ to $5 \mathrm{mM}$ at an increment of $1 \mathrm{mM}$. (a) the electrode A; sensing layer only (black line), (b) the electrode B1; the sensor with the overcoated layer (red line), (c) the electrode B2; the sensor with the twice amount of the overcoated layer than B1 (blue line), as described in the text. 


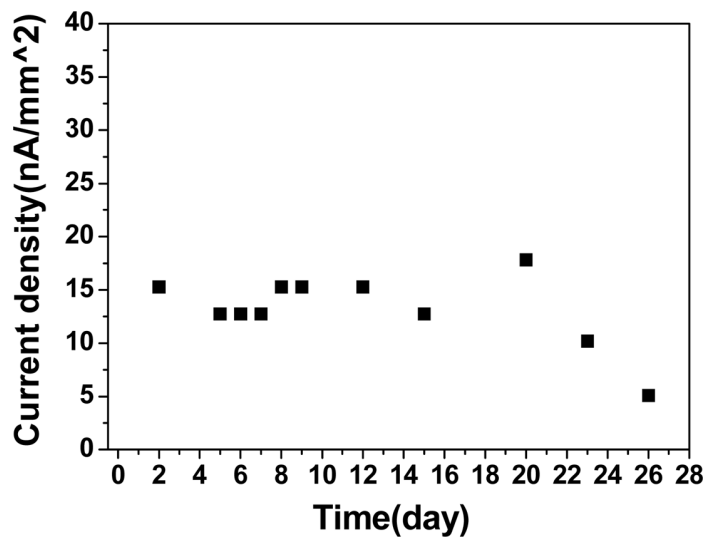

Fig. 4. Current density of $\mathrm{B} 2$ electrode for $5 \mathrm{mM}$ glucose in $\mathrm{PBS}$ at $0.35 \mathrm{~V}$ vs. $\mathrm{Ag} / \mathrm{AgCl}$.

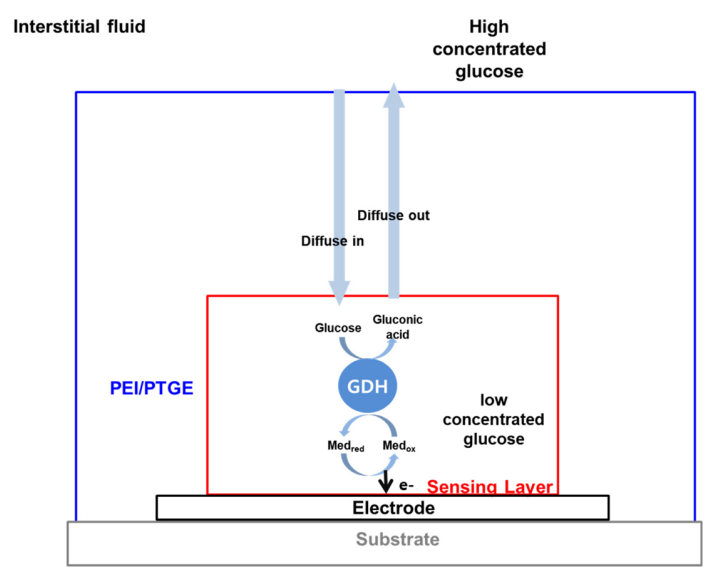

Fig. 5. Scheme of the electrochemical reactions and ion/ mass transport in the glucose sensor having overcoating layer of PEI/PTGE.

ing no coating layer (the electrode A) was tested, and it was found that the signal decreased significantly in 1 day (data not shown). It confirms the coating layer is essential for the long-term use of the sensor in the body. The current response of the electrode $\mathrm{B}$ is $15 \mathrm{nA} / \mathrm{mm}^{2}$ for $5 \mathrm{mM}$ glucose concetration (Fig. 4), and the current range can be calculated to be $0.6 \sim$ $18 \mathrm{nA}$, when the electrode area is $0.2 \mathrm{~mm}^{2}$. Therefore, the coating layer enables to measure the glucose in the body fluid from $1 \mathrm{mM}$ to $30 \mathrm{mM}$,

In summary, the structure of the sensor and the transport of the molecules can be depicted, as shown in Fig. 5. The PEI/PTGE layer is located outside of the sensing layer to reduce the diffusion of glucose.
The electrocatalytic reactions occur in the sensing layer, which is a redox hydrogel containing the GDH and redox polymers, and the following transport of substrate and ions occurring in the sensing layer is limited by the transport in the overcoating layer. Then, the rate-limiting factor, which is given by the current measured, is the transport through the overcoating layer. The newly developed PEI/PTGE coating layer shows the promise for applying to the glucose sensor in CGMS. It is simple in preparation, has appropriate current and response ranges, and it operates long-term stable enough for the CGM sensor.

\section{Conclusions}

The newly-developed PEI/PTGE layer was introduced to stabilize the glucose for the continuous glucose monitoring system. The simple drop-coating of the overlayer could reduce the glucose response down to $1 / 50$, but still, the glucose response was linear at this condition. The sensor was stable over 20 days for the continuous measurement of the glucose in the PBS solution. The calculated response is $0.6 \sim$ $18 \mathrm{nA}$ for $1 \mathrm{mM}$ to $30 \mathrm{mM}$ glucose when the sensor is fabricated to have an area of $0.2 \mathrm{~mm}^{2}$. It shows the promise to be applied to measure the glucose in the body fluid continuously.

\section{Acknowledgment}

This research was supported by the Next-generation Medical Device Development Program for Newly-Created Market of the National Research Foundation (NRF) funded by the Korean government, MSIP (No. 2015M3D5A1065759).

\section{References}

[1] D. Rodbard, Diabetes Technol. Ther. 2016, 18(S2), S2-3.

[2] Z.Mian, K. L. Hermayer and A. Jenkins, Am. J. Med. Sci. 2019, 358(5), 332-339.

[3] S. Vashist, Diagnostics 2013, 3(4), 385-412.

[4] A. Facchinetti, G. Sparacino and C. Cobelli, J. Diabetes Sci. Technol. 2007, 617-623.

[5] D. B. Keenan, J. J. Mastrototaro, G. Voskanyan and G. M. Steil, J. Diabetes Sci. Technol. 2009, 3(5), 12071214.

[6] A. Heller and B. Feldman, Acc. Chem. Res. 2010, 43(7), 963-973.

[7] R. Sternberg, D. S. Bindra, G. S Wilson and D. R. Thévenot, Anal. Chem., 1988, 60(24), 2781-2786. 
[8] T. Kulkarni and G. Slaughter, Membrane(Basel), 2016, $6(4), 55$

[9] I. Becerik, Ş. Süzer and F. J. Kadirgan, Electroanal. Chem., 1999, 476(2), 171-176.

[10] A. Fraleoni-Morgera, B. Ballarin, A. Filippini, D. Frascaro, C. Piana and L.Setti, Biosens. Bioelectron. 2005, 20(10), 2019-2026

[11] M. H. Freeman, J. R. Hall and M. C. Leopold, Anal. Chem., 2013, 85(8), 4057-4065.

[12] H. Jo, H. Piao and Y. Son, J. Electrochem. Sci. Technol. 2013, 4(1), 41-45.

[13] D. J. Harrison, R. F. B. Turner and H. P. Baltes, Anal. Chem., 1988, 60(19), 2002-2007.

[14] C. J. Yuan, C. L. Hsu, S. C. Wang and K. S. Chang, Electroanalysis 2005, 17(24), 2239-2245.

[15] O. M. Schuvailo, O. O. Soldatkin, A. Lefebvre, R. Cespuglio and A. P. Soldatkin, Anal. Chim. Acta 2006, 573, 110-116.

[16] J. H. Han, J. D. taylor, D. S. Kim, Y. S. Kim, Y. T. Kim, G. S. Cha and H. Nam, Sensors Actuators, B Chem.,
2007, 123(1), 384-390.

[17] B. Yu, N. Long, Y. Moussy and F. Moussy, Biosens. Bioelectron. 2006, 21(12), 2275-2282.

[18] A. Koh, D. A. Riccio, A. W. Carpenter, S. P. Nichols and M. H. Schoenfisch, Biosens. Bioelectron. 2011, 28(1), 17-24.

[19] J. E. Song, Z. Hong, R. K. Nagarale and W. S. Shin, J. Electrochem. Sci. Technol. 2011, 2(3), 163-167.

[20] X. Zeng, and E. Ruckenstein, J. Memb. Sci., 1998, 148(2), 195-205.

[21] M. M. Rahman and J. J. Lee, J. Electrochem. Sci. Technol. 2019, 10(2), 185-195.

[22] H. D. Jirimali, D. Saravanakumar and W. Shin, J. Electrochem. Sci. Technol. 2018, 9(3), 169-175.

[23] T. J. Ohara, R. Rajagopalan and A. Heller, Anal. Chem. 1993, 65(23), 3512-3517.

[24] S.-J. Kim, Y. Quan, E. Ha and W. S. Shin, J. Electrochem. Sci. Technol. 2021, 12(1), 33-37.

[25] Q. Gao and X. Yang, Chem. Commun. 2004, 1, 30-31. 